\title{
INTERFACIAL MICROSTRUCTURE OF Ni/Si-BASED OHMIC CONTACTS TO GaN
}

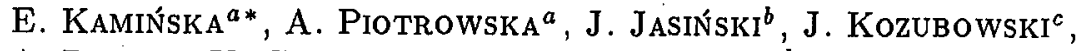 \\ A. BARCZ ${ }^{a}$, K. GolASZEWSKA ${ }^{a}$, M.D. BREMSER ${ }^{d}$ AND R.F. DAVIS ${ }^{e}$ \\ ${ }^{a}$ Institute of Electron Technology, Al. Lotników 32/46, 02-668 Warsaw, Poland \\ ${ }^{b}$ Institute of Experimental Physics, Warsaw University, Warsaw, Poland \\ ${ }^{c}$ Faculty of Mat. Science and Engineering, Warsaw Technical University \\ Warsaw, Poland \\ ${ }^{d}$ Aixtron AG, Aachen, Germany \\ ${ }^{e}$ Department of Mat. Science and Eng., North Carolina State University, Raleigh, USA
}

The microstructure of Ni/Si-based contacts to $\mathrm{GaN}$ has been studied using transmission electron microscopy methods. The transition from non-ohmic to ohmic behavior appears to correlate with the initial limited reaction of $\mathrm{GaN}$ with $\mathrm{Ni}$ and further $\mathrm{Si}-\mathrm{Ni}$ reaction-driven decomposition of the interfacial $\mathrm{GaN}-\mathrm{Ni}$ phase.

PACS numbers: 73.40.Cg, 73.40.Ns

Development of low-resistance, thermally stable ohmic contacts is one of the big challenges for the progress in the GaN device technology. We have recently reported on novel $\mathrm{Ni} / \mathrm{Si}$-based metallization systems providing ohmic contacts on GaN [1]. We have adopted the idea of Sands et al. [2] of the incorporation of a dopant into the semiconductor subcontact region by solid-phase dissolution and regrowth (SPR). To produce contacts to $p$-type $\mathrm{GaN}, \mathrm{Mg}$ dopant is added to the $\mathrm{Ni} / \mathrm{Si}$ metallization; for $n$-type $\mathrm{GaN}, \mathrm{Si}$ is the intended donor dopant [1]. We have shown that these contacts exhibit ohmic behavior after annealing at temperatures ranging from 400 to $600^{\circ} \mathrm{C}$, with resistivities of about $1 \times 10^{-3} \Omega \mathrm{cm}^{2}$ at $p$-type $\left(p \approx 3 \times 10^{17} \mathrm{~cm}^{-3}\right)$ and $n$-type $\left(n \approx 2 \times 10^{17} \mathrm{~cm}^{-3}\right)$ GaN. The preliminary characterization using Rutherford backscattering spectrometry (RBS), secondary ion mass spectrometry (SIMS), and X-ray diffraction (XRD) indicated that several thermally activated solid-phase processes take place during the formation of ohmic contacts. However, a full understanding of these processes and their influence on the metal/GaN interface properties is still lacking.

This study will focus on microstructure of $\mathrm{Ni} / \mathrm{Si}$-based contacts. In order to investigate structural properties such as interface abruptness, phase composition, and defects in the contact region we have applied cross-sectional transmission

*e-mail: eliana@ite.waw.pl 
electron microscopy (XTEM) and high resolution electron microscopy (HREM) combined with energy dispersive X-ray spectroscopy (EDX). The high spatial resolution of electron microscopy can provide much more details about the contact structure and thus enable a better understanding of the results previously obtained using other techniques.

The GaN epilayers were grown on AlN buffer layer on $6 \mathrm{H}-\mathrm{SiC}(0001)$ substrates via organometallic vapor phase epitaxy [3]. The metallization in a form of sandwich structures $\mathrm{Ni} / \mathrm{Mg} / \mathrm{Ni} / \mathrm{Si}$ and $\mathrm{Ni} / \mathrm{Si}$ for $p$ - and $n$-type GaN, respectively, was deposited by e-beam evaporation. Formation of ohmic contacts was completed by heat treatment in flowing $\mathrm{N}_{2}$.

TEM observations were performed using a JEOL JEM 3010 microscope operating at $300 \mathrm{keV}$. A microprobe beam size of $c a .10 \mathrm{~nm}$ was used for EDX analysis. XTEM specimens were prepared by polishing and dimpling, followed by $\mathrm{Ar}^{+}$ion milling. The interpretations of the EDX data and selected-area diffraction (SAD) patterns have been checked by comparison with the composition estimated using RBS and SIMS, and the phases identified by XRD.
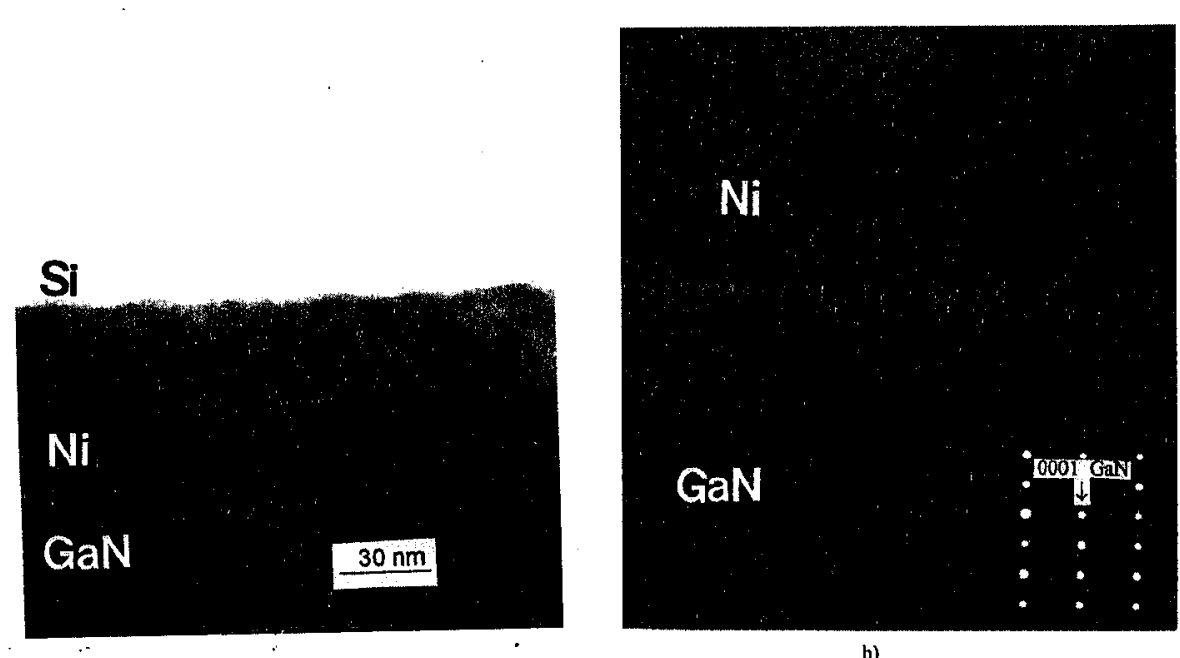

b)

Fig. 1. (a) XTEM image of the as-deposited $\mathrm{GaN} / \mathrm{Ni} / \mathrm{Si}$ contact, (b) HREM lattice image of the interface of the as-deposited $\mathrm{GaN} / \mathrm{Ni} / \mathrm{Si}$ contact.

Figure 1a shows a TEM micrograph of the cross-section of the as-deposited contact. The contact consists of three distinct layers. The upper Si layer is amorphous, the intermediate $\mathrm{Ni}$ film is polycrystalline. No sign of reaction at the $\mathrm{Si} / \mathrm{Ni}$ interface was observed. The high resolution image of the contact interface, shown in Fig. 1b, revealed that the first layer, about $2 \mathrm{~nm}$ thick, adjacent to GaN, exhibits a crystalline structure. This layer, too thin to resolve separately by EDX analysis, is laterally continuous along the entire interface. One might expect the interfacial layer to be the product of the interaction between Ni and GaN. In fact, a limited $\mathrm{GaN}-\mathrm{Ni}$ interaction was revealed by SIMS and XRD analysis [1]. SIMS 
depth profiling indicated that both $\mathrm{Ga}$ and $\mathrm{N}$ accumulate at the contact interface, while with the use of $\mathrm{X}$-ray diffraction analysis $\mathrm{Ni}_{3} \mathrm{Ga}_{2}$ phase was identified in the as-deposited GaN/Ni contact. The lack of nitrogen-related phases implies that nitrogen is atomically dispersed in the interfacial layer, as already suggested by Bermudez et al. [4]. Here, we were unable to conclusively identify the composition of the interfacial layer in the as-deposited contact, but the distance between lattice fringes taken from the HREM image $d=0.202 \mathrm{~nm}$ is almost identical to that of the hexagonal $\mathrm{Ni}_{3} \mathrm{Ga}_{2}$ phase $d_{(11 \overline{2} 0)}=0.2000 \mathrm{~nm}$ [5]. No interfacial oxide was observed.
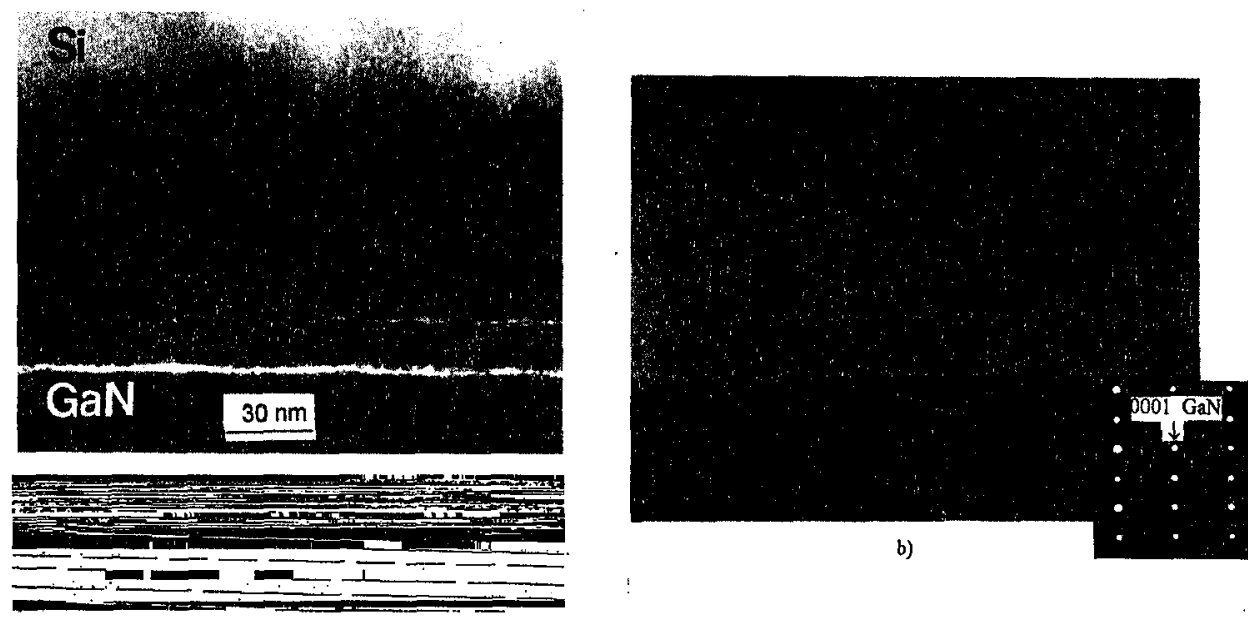

Fig. 2. (a) XTEM image of the $\mathrm{GaN} / \mathrm{Ni} / \mathrm{Si}$ contact annealed at $500^{\circ} \mathrm{C}$, (b) $\mathrm{HREM}$ lattice image of the interface of the $\mathrm{GaN} / \mathrm{Ni} / \mathrm{Si}$ contact annealed at $500^{\circ} \mathrm{C}$.

The microstructural changes in the contact region after annealing at $500^{\circ} \mathrm{C}$ for $30 \mathrm{~min}$ to achieve the ohmic behavior are presented in Fig. 2. The $\mathrm{Ni}_{3} \mathrm{Ga}_{2}$ phase has decomposed. Ni liberated from that phase together with unreacted Ni have been entirely consumed by Si to form nickel silicide NiSi. An excess of unreacted amorphous Si was detected in the outermost layer. Along the interface separating the semiconductor and the metallization, a 10-12 nm thick amorphous planar interlayer formed. The careful EDX analysis revealed $\mathrm{Si}$ as the only component of this layer. The high resolution imaging of the interface, Fig. 2b, revealed crystalline inclusions adjacent to the GaN surface. Fourier transform of the lattice fringes taken from these HREM images indicates that these inclusions exhibit a cubic structure with the lattice parameter $a=0.52 \mathrm{~nm}$, which, within the resolution of our measurement, could suggest $\mathrm{Si}(a=0.5431 \mathrm{~nm}[6])$. The contact interface is sharp and abrupt.

The transition from non-ohmic to ohmic behavior in $\mathrm{GaN} / \mathrm{Ni} / \mathrm{Si}$ contact system takes place when the initial interface interlayer of $\mathrm{Ni}_{3} \mathrm{Ga}_{2}: \mathrm{N}$ decomposes as a result of $\mathrm{Si}-\mathrm{Ni}$ reaction. This process is certainly accompanied by a regrowth of the decomposed volume of the semiconductor. Since the semiconductor surface layer reacted with $\mathrm{Ni}$ is very thin, the observed ohmic behavior must be due to a few monolayers thick regrown GaN film. The growth of the Si layer at the surface of GaN could lower the barrier height across the Si/GaN interface, and thus fa- 
cilitate the formation of the low-resistance contact. The interface of $\mathrm{Ni} / \mathrm{Si}$-based contacts to GaN appears to be sharp as opposed to a continuous diffuse interface, characteristic of the presently used alloyed contacts. In terms of electronic properties, interface and surface morphologies, and long term stability, the $\mathrm{Ni} / \mathrm{Si}$ contact scheme appears to be promising for device applications.

The authors acknowledge Piotr Bogusławski for valuable discussions. This work was partially supported by the Committee for Scientific Research (Poland) grant No. PBZ 28.11/P9.

\section{References}

[1] E. Kamińska, A. Piotrowska, A. Barcz, M. Guziewicz, S. Kasjaniuk, M.D. Bremser, R.F. Davis, E. Dynowska, S. Kwiatkowski, Mater. Res. Soc. Symp. Proc. 482, 1077 (1998).

[2] T. Sands, E.D. Marshall, L.C. Wang, J. Mater. Res. 3, 914 (1988).

[3] T.W. Weaks, Jr., M.D. Bremser, K.S. Ailey, E. Carlson, W.G. Perry, E.L. Piner, N.A. El-Masry, R.F. Davis, J. Mater. Res. 10, 1011 (1996).

[4] V.M. Bermudez, R. Kaplan, M.A. Khan, J.N. Kuznia, Phys. Rev. B 48, 2436 (1993).

[5] JCPDS Card No. 7-150.

[6] JCPDS Card No. 27-1402. 